\title{
Comparative Analysis of Pso-Pid and Hu-Pid
}

\author{
Chanda Thakur, Navdeep Batish \\ Power system-M.tech(Scholar) I.K.G Punjab Technical University Associate Prof.-S.S.C.E.T.
}

\begin{abstract}
PID control is an important ingredient of a distributed control system. The controllers are also embedded in many special purpose control systems. PID control is often combined with logic, sequential functions, selectors, and simple function blocks to build the complicated automation systems used for energy production, transportation, and manufacturing. Many sophisticated control strategies, such as model predictive control, are also organized hierarchically. PID control is used at the lowest level; the multivariable controller gives the set points to the controllers at the lower level. The PID controller can thus be said to be the "bread and butter' of power system engineering. It is an important component in every control engineer's tool box. PID controllers have survived many changes in technology, from mechanics and pneumatics to microprocessors via electronic tubes, transistors, integrated circuits. The microprocessor has had a dramatic influence on the PID controller
\end{abstract}

Keywords: Include at least 5 keywords or phrases

\section{INTRODUCTION}

The PID controller is the most common form of feedback. It was an essential element of early governors and it became the standard tool when process control emerged in the 1940s. In process control today, more than $95 \%$ of the control loops are of PID type, most loops are actually PI control. PID controllers are today found in all areas where control is used. The controllers come in many different forms. There are standalone systems in boxes for one or a few loops, which are manufactured by thousands yearly. PID control is an important ingredient of a distributed control system. The controllers are also embedded in many special purpose control systems [15]. PID control is often combined with logic, sequential functions, selectors, and simple function blocks to build the complicated automation systems used for energy production, transportation, and manufacturing. Many sophisticated control strategies, such as model predictive control, are also organized hierarchically. PID control is used at the lowest level; the multivariable controller gives the set points to the controllers at the lower level. The PID controller can thus be said to be the "bread and butter' of control engineering. It is an important component in every control engineer's tool box. PID controllers have survived many changes in technology, from mechanics and pneumatics to microprocessors via electronic tubes, transistors, integrated circuits. The microprocessor has had a dramatic influence on the PID controller [16]. In general it can be said that $\mathrm{P}$ controller cannot stabilize higher order processes. For the 1st order processes, meaning the processes with one energy storage, a large increase in gain can be tolerated. Proportional controller can stabilize only 1 st order unstable process. Changing controller gain $\mathrm{K}$ can change closed loop dynamics. A large controller gain will result in control system with: Smaller steady state error, i.e. better reference following, faster dynamics, i.e. broader signal frequency band of the closed loop system and larger sensitivity with respect to measuring noise and smaller amplitude and phase margin. When $\mathrm{P}$ controller is used, large gain is needed to improve steady state error. Stable systems do not have problems when large gain is used. Such systems are systems with one energy storage (1st order capacitive systems). If constant steady state error can be accepted with such processes, than $\mathrm{P}$ controller can be used. Small steady state errors can be accepted if sensor will give measured value with error or if importance of measured value is not too great anyway.

D mode is used when prediction of the error can improve control or when it necessary to stabilize the system. From the frequency characteristic of $D$ element it can be seen that it has phase lead of $90^{\circ}$. Often derivative is not taken from the error signal but from the system output variable. This is done to avoid effects of the sudden change of the reference input that will cause sudden change in the value of error signal. Sudden change in error signal will cause sudden change in control output. To avoid that it is suitable to design $\mathrm{D}$ mode to be proportional to the change of the output variable. PD controller is often used in control of moving objects such are flying and underwater vehicles, ships, rockets etc. One of the reason is in stabilizing effect of PD controller on sudden changes in heading variable $\mathrm{y}(\mathrm{t})$. Often a "rate gyro" for velocity measurement is used as sensor of heading change 
of moving object. PI controller will eliminate forced oscillations and steady state error resulting in operation of on-off controller and $\mathrm{P}$ controller respectively. However, introducing integral mode has a negative effect on speed of the response and overall stability of the system. Thus, PI controller will not increase the speed of response. It can be expected since PI controller does not have means to predict what will happen with the error in near future. This problem can be solved by introducing derivative mode which has ability to predict what will happen with the error in near future and thus to decrease a reaction time of the controller. PI controllers are very often used in industry, especially when speed of the response is not an issue. A control without D mode is used when: Fast response of the system is not required, large disturbances and noise are present during operation of the process ,there is only one energy storage in process (capacitive or inductive), there are large transport delays in the system.

Process control has become increasingly important in the process industries as a consequence of global competition, rapidly changing economic conditions, and more stringent environmental and safety regulations. Process control is also a critical concern in the development of more flexible and more flexible and more complex processes for manufacturing high value added products. Any study of process control must begin by investigating the concept of a process. It is generally thought of as a place where materials and most often, energy come together to produce a desired product. From a control viewpoint the meaning is more specific. A process is identified as leaving one or more variables associated with it that are important enough for their values to be known and for them to be controlled. One of the complex and difficult in process control ids control tuning. Control tuning is the major key issue to operate the plant. Process tuning is a key role in ensuring that the plant performance satisfies the operating objectives. Controller tuning inevitably involves a tradeoff between performance and robustness. The performance goals of excellent set-point tracking and disturbance rejection should be balanced against the robustness goal of stable operation over a wide range of conditions.

The Process control system is the entity that is charged with the responsibility for monitoring outputs, making decisions about how best to manipulate inputs so as to obtain desired output behavior, and effectively implement such decisions on the process [3]. Control systems are classified into two general categories open loop and closed loop control systems. Open loop control systems are control systems in which the output has no effect upon the control action. In an open-loop control system, the output is neither measured nor fed back for comparison with the input. For example, in a washing machine, soaking, washing, and rinsing are operated on a time basis. The machine does not measure the output signal namely the cleanliness of clothes. In any open-loop control system the output is not compared with the reference input. Hence, for each reference input, there corresponds a fixed operation condition. Thus, the accuracy of the system depends on the calibration. In the presence of disturbance, an openloop control system will not confirm the desired task. Open-loop control can be used in practice only if the relationship between the input and output is known and if there are neither internal nor external disturbances. Clearly such systems are not feedback control systems. Any control system, which operates on a time basis, is open loop. Advantages of open loop control systems are simple construction, very much convenient when output is difficult to measure, and such systems are easy from maintenance point of view. Generally, these are free from the problems of stability. Such systems are simple to design, economical and give inaccurate results if there are variations in the external environment i.e. they cannot sense environmental changes. They are inaccurate and unreliable because accuracy of such system is totally dependent on the accurate pre-calibration of the controller. Similarly they cannot sense internal disturbances in the system, after the controller stage. To maintain the quality and accuracy, recalibration of the controller is necessary from time-to-time. To overcome all the above disadvantages, generally closed loop systems are used in practice.

Closed loop control system is one in which the output signal has a direct effect upon the control action. That is, a closed-loop control system incorporates feedback element. The actuating error signal, which is the difference between the input signal and the feedback signal (which may be the output signal or function of the output signal and its derivatives), is fed to the controller so as to reduce the error. In other words, the term 'closed loop' implies the use of feedback action in order to reduce the system error. The error signal produced in the automatic controller is amplified and the output of the controller is sent to the control value in order to change the valve opening for steam supply so as to correct the actual water temperature. If there is no error, no change in the valve operation is necessary. The control of a complex system by a human operation is not effective because of the many interrelations among various variables. Automatic control systems eliminate any human error in operation. If high precision control is necessary, control must be automatic. System in which the controlling action or input is somehow 
dependent on the output or changes in output are called closed loop system. Feedback is a property of the system by which it permits the output to be compared with the reference input so that appropriate controlling action can be decided. In such a system, output or part of the output fed back to the input for comparison with the reference input applied to it. It is not possible in all the systems that available signal can be applied as input to the system. Depending upon the nature of controller it is required to reduce it or amplify to change its nature. This changed input as per requirement is called reference input, which is to be generated by using reference transducer. The main excitation to make the system called its command input, which is then applied to the reference transducer to generate reference input. The output, which is to be decided by feedback element, is fed back to the reference input. The signal, which is output of feedback element, is called 'feedback signal' $b(t)$. It is then compared with the reference input giving error signal $\mathrm{e}(\mathrm{t})=\mathrm{r}(\mathrm{t})+\mathrm{b}(\mathrm{t})$. When feedback signal is positive it is called positive feedback system and if the signal is negative it is called negative feedback system. This modified error signal then actuates the actual system and produces the controlled output $c(t)$.

An advantage of closed loop control system is that the use of feedback makes the system response relatively insensitive to external disturbances and internal variations in the system parameters. It is thus possible to use relatively inaccurate and inexpensive components to obtain the accurate control of a given plant, whereas this is impossible in the open-loop case. From the point of ability, the open-loop control system is easier to build since stability is not a major problem. On the other hand, stability is always a major problem in the closed-loop control system since it may tend to over correct errors, which may cause oscillations of constant or changing amplitude. It should be emphasized that for systems in which the inputs are known ahead of time and in which there are no disturbances, it is advisable to use open-loop control. Closed-loop control systems have advantages only when unpredictable disturbances and/or unpredictable variations in system components are present. A proper combination of open-loop and closed-loop control is usually less expensive and satisfies the overall system performance. The design and implementation of smart structural systems necessitates the integration of mechanical systems with sensors, actuators, and control systems for higher performance and selfdiagnosis capabilities. A key element of this combination is the integration of the control system into the structure [4]. The process control system is the entity that is charged with the responsibility for monitoring outputs, making decisions about how best to manipulate inputs so as to obtain desired output behavior, and effectively implement such decisions on the process [5]. The process has a property called self-regulation. A self- regulating system does not provide regulation of a variable to any particular reference value. In process control, the basic objective is to regulate the value of some quantity. To regulate means to maintain that quantity at some desired value regardless of external influences. The desired value is called the reference value or set point. In many industrial process control systems, the control process is complex in mechanism, and varying with time. So, general PID control is very difficult to obtain satisfactory effects because it is not self-adaptive for many varying factors such as parameter varying.

The process dynamics are concerned with analyzing the dynamic (i.e, time dependent) behavior of a process in response to various types of inputs. In other words, it is the behavior of a process as time progresses [6, 7]. A process is a progressively continuing operation that concedes of a series of controlled actions or movements systematically directed towards a particular result or end. When the automatic control is applied to system, which is designed to regulate the value of some variable to a set point, it is called process control. Examples are chemical, economic, and biological processes. An automatic regulation system in which the output is a variable such as temperature, pressure, flow, liquid level, or $\mathrm{pH}$ is called a process control system. Process control is widely applied in industry. Programmed control such as the temperature control of heating furnaces in which the furnace temperature is controlled according to preset program is often used in such systems. For example, a preset program may be such that the furnace temperature is raised to a given temperature in some given time interval and then lower to another given temperature in some other given time interval. In such program control, the set point is varied according to the present time schedule. The controller then functions to maintain the furnace temperature close to the varying set point. It should be noted that most process control systems include servomechanism as an integral part [8].Process engineers are often responsible for the operation of chemical processes. As these processes become larger scale and/or more complex, the role of process automation becomes more and more important. The objective of this textbook is to teach process engineers how to design and tune feedback controllers for the automated operation of chemical processes. 


\section{LITERATURE REVIEW}

This section covers the research findings related to tuning of industrial processes through different tuning algorithm. There are number of control strategies and methods for controlling concentration and temperature of process model which have been implemented by researchers from all over the world. The formulation of variety objective of overcoming the nonlinearity problem by having a robust and effective controller as compared to the conventional techniques.

A.Jayachitra [1] Genetic algorithm (GA) based PID (proportional integral derivative) controller has been proposed for tuning optimized PID parameters in a continuous stirred tank reactor (CSTR) process using a weighted combination of objective functions, namely, integral square error (ISE), integral absolute error (IAE), and integrated time absolute error (ITAE). Optimization of PID controller parameters is the key goal in chemical and biochemical industries. PID controllers have narrowed down the operating range of processes with dynamic nonlinearity. In this work, globally optimized PID parameters tend to operate the CSTR process in its entire operating range to over come the limitations of the linear PID controller. The simulation study reveals that the GA based PID controller tuned with fixed PID parameters provides satisfactory performance in terms of set point tracking and disturbance rejection..

Wen Tan [2]the author compared the well-known PID tuning rules. Criteria based on disturbance rejection and system robustness are proposed to assess the performance of PID controllers. A simple robustness measure is defined and the integral gains of the PID controllers are shown to be a good measure for disturbance rejection. The integral error is generally accepted as a good measure for system performance. Clearly, if the response is critically damped, IE will be equal to IAE. However, if it is weakly damped, then IE will not be suitable as a performance measure.

K. J. Åström et al. [3] analyzed the Ziegler-Nichols step response method for PID control. The Ziegler-Nichols step response method is based on the idea of tuning controllers based on simple features of the step response. This paper has revisited tuning of PID controllers based on step response experiments in the spirit of Ziegler and Nichols. Ziegler and Nichols developed their tuning rules by simulating a large number of different processes, and correlating the controller parameters with features of the step response. Process dynamics was characterized by the parameters obtained from the step response. A nice feature of this design method is that it permits a clear tradeoff between robustness and performance. The idea is investigated from the point of view of robust loop shaping. The results are insight into the properties of PI and PID control and simple tuning rules that give robust performance for processes with essentially monotone step responses.

Geetha, M., Manikandan [4] the author presents the optimal design of PID controller based on a particle swarm optimization (PSO) approach for continuous stirred tank reactor (CSTR). The mathematical model of experimental system had been approximate near the operating point for the PSO algorithm to adjust PID parameters for the minimum integral of time multiplied by absolute error (ITAE) condition. This research explains a design of PID controller by using the PSO method to search for optimal parameters converting into the optimal point and the good control response based on the optimal values by the PSO technique.

S. Palanki et al. [5] developed software module to run a simulation via the internet. The software module is developed in MATLAB and simulates a regulation problem in a continuous stirred tank reactor (CSTR) in which a series reaction is occurring. The user has the option to input a wide variety of system parameters, initial conditions, final time, and controller parameters. The effect of changing these values on the overall system dynamics can be studied easily. The development of such modules eliminates space, time, and cost constraints. It was found that this software module was a useful teaching supplement to the traditional classroom lecture. Students were able to study the effect of changing various process parameters as well as controller parameters on the regular output. The interactive distance learning concepts include the use of remote computer access to enhance self-paced learning. The internet provides a real-time link that eliminates space time constraints, and gives access from anywhere at any time. Moreover, due to the multiuser-multitasking nature of computer environments, several students can run the software module at the same time. The development of a virtual laboratory has the potential to deliver experiences which are not accessible to students in the real world. Recent technological advances in computer software are bringing virtual laboratories within the reach of educational and student budgets.

J. Kennedy et al. [6] introduced particle swarm methodology for the optimization of nonlinear functions. Particle swarm optimization is an extremely simple algorithm that seems to be effective for optimizing a wide range of functions. We view it as a biologically derived algorithm, occupying the space in nature between evolutionary searches, which occurs on the order of milliseconds. Particle swarm optimization as developed by authors comprises a very simple concept, and paradigms can be implemented in a few lines of 
computer code. It requires only primitive mathematical operators, and is computationally inexpensive in terms of both memory requirements and speed. Early testing has found the implementation to be effective with several kind of problems. This paper discusses application of the algorithm to the training of artificial neural network weights. Particle swarm optimization has also been demonstrated to perform well on genetic algorithm test functions. The adjustment toward pbest and gbest by the particle swarm optimizer is conceptually similar to the crossover operation utilized by genetic algorithms.

B. Nagaraj et al. [7] compared the different soft computing techniques for PID controller. The methodology and efficiency of the proposed method are compared with that of traditional methods. Determination or tuning of the PID parameters continues to be important as these parameters have a great influence on the stability and performance of the control system. Research work has been carried out to get an optimal PID tuning by using GA, EP, PSO and ACO. The results obtained reflect that use of soft computing based controller improves the performance of process in terms of time domain specifications, set point tracking, and regulatory changes and also provides and optimum stability.

Parvesh Saini et al. [8] The Continuous stirred tank reactor is most deciding section chemical processes based industries. It is necessary to have to have the proper analysis of CSTR in chemical industries for obtaining the desire product. The two controllers are present in this paper (Fuzzy logic Controller (FLC), Proportional-IntegralDerivative(PID) are designed to control the CSTR temperature and a comparative analysis of the controllers have been presented. In the presence of step disturbance the behavior of CSTR has been tested them and then the control methodologies have been developed to control the temperature. In the chemical process insutries the CSTR shows the extremely non-linear behavior and generally has varied operating range.

Bikas Mondal et al. [9] in many industrial applications conventional controller are used due to their simplicity and robustness. All the systems are non-linear in nature: Conventional controller is not always providing good and correct result. So for better response Fuzzy Logic Controller are used. IN this paper to compare the control effect due to PID control and Fuzzy control. When comparing the fuzzy control and PID control will see that fuzzy control is superior then the PID control. More concentration is provided by fuzzy control to different parameters, such as the response time, the steady state error and overshoot. The paper work indicates that in triangular membership function and centroid defuzzification method the fuzzy logic controller application gives the best response. The PID controller are easy to implement and also easy to understand in hardware and software, and very simple and a process model is not required for operation and initialization. Conventional PID is not very efficient due to the presence of nonlinearity. In various industrial processes Fuzzy Logic Controller (FLC) are used for taking proper action likes human control actions.

G. Reynoso-Meza et al. [10] For the several industrial processes Proportional - Integral Derivative (PID) controllers remains as reliable and practical control solutions. The major merit of the PID controller is they are easy to implement and gives a better trade-off between simplicity and cost of implement. In this paper for unstable process propose a multi-objective optimization design , using PID controller. The procedure for this kind of process, sometimes difficult to control; comparison with existing tuning rule methods provide promising results for this tuning procedure. For the fulfilment of the several objectives and requirements new techniques are being focused, sometimes in conflict among them. For controller tuning applications recently a new technique is have been shown that is Multiobjective Optimization (MOO). The Multiobjective Optimization technique unable the decision maker and designer having having a close embedment into the tuning process since it is possible to take into account each design objective individually; the comparing design alternatives are also enable by this technique(that is different controllers), in order to select a tuning fulfilling the expected trade-off among conflicting objectives. In the open loop stable systems efforts are particularly concentrated; nevertheless some critical processes as continuous stirred tank reactors and bioreactors, common in chemical processing units and biological processes, are unstable open loop systems. For such process; nevertheless, efforts to merge multi-objective optimization techniques have been not yet applied for such instances PID- like controller tuning have been main focus in work.

J.C. Basilio et al. [11] proposed methodologies for tuning PI and PID controllers. Like the well-known Ziegler- Nichols method, they are based on the plant step response. The methodology also encompasses the design of PID controllers for plants with under damped step response and provides the means for a systematic adjustment of the controller gain in order to meet transient performance specifications. Unlike the Ziegler-Nichols step response method, they provide systematic means to adjust the proportional gain in order to have no overshoot on the closed-loop step response. In addition, since all the development of 
the methodology relies solely on concepts introduced in a frequency-domain-based control course, the paper has also a didactic contribution

\section{CONCLUSIONS}

The proposed PSO-PID and HU-PID controller is tested by using Mat-lab Simulink program and their performance is compared. A HUPID controller is best implemented. Also, the PID controller parameters obtained from HU algorithm gives better tuning result as compared to PSO-PID rule. The major impact of $\mathrm{HU}$ is on integral square error and peak overshooting. Both are minimized by HU-PID controller. HU-PID is a very simple concept, and paradigms can be implemented in a few lines of computer code.

\section{ENCES}

\section{REFER}

[1]. Jayachitra, A., and R. Vinodha. "Genetic algorithm based PID controller tuning approach for continuous stirred tank reactor." Advances in Artificial Intelligence, vol 12, 2014 (2014):9.

[2]. Zribi, Ali, Mohamed Chtourou, and Mohamed Djemel. "A New PID Neural Network Controller Design for Nonlinear Processes." arXiv preprint arXiv:1512.07529, vol 5, (2015).

[3]. Mohanty, Asit, Meera Viswavandya, and Sthitapragyan Mohanty. "An optimised FOPID controller for dynamic voltage stability and reactive power management in a stand-alone micro grid." International Journal of Electrical Power \& Energy Systems vol 78 (2016): 524-536.

[4]. Geetha, M., Manikandan, P., Shanmugapriya, P., Silambarasan, V., \& Naveen, R. (2013, July). Real-time implementation and performance analysis of two dimension PID fuzzy controller for continuous stirred tank reactor. In Computing, Communications and Networking Technologies (ICCCNT), 2013 Fourth International Conference on (pp. 1-5, vol 2). IEEE.

[5]. Perng, Jau-Woei, Guan-Yan Chen, and YaWen Hsu. "FOPID controller optimization based on SIWPSO-RBFNN algorithm for fractional-order time delay systems." Soft Computing (2016): 1-14.

[6]. K. J. Astrom and T. Hagglund, "Revisiting the Ziegler-Nichols step response method for PID control," Journal of Process control, vol. 14, pp. 635-650,2004.

[7]. Smith C.A, and Corripio, A.B, "Principles and Practice of Automatic Process Control:, John Wiley and Sons, 1985.Kastauhiko
Ogata, "Modern control engineering", PIII, Twenty-third edition-1990.

[8]. J.C. Basilio and S. R. Matos, "Design of PI and PID controllers with transient performance specification," IEEE Trans. on Education, vol. 45, no. 4, pp. 364-370,2002.

[9]. Wen Tan, Jizhen Liu, Tongwen Chen and Horaciao J. Marquez, "Comparision of some well known PID tuning formulas," Trans. on Computers and Chemical Engineering, vol. 30, pp. 1416-1423,2006.

[10]. K. J. Åström and T. Hägglund, "Revisiting the Ziegler-Nichols step response method for PID control," Journal of Process control, vol. 14, pp. 635-650,2004.

[11]. Mohammad Ali Nekoui, Mohammad Ali Khameneh and Mohammad Hosein Kazemi,"'Optimal design of PID controller for a CSTR system using Particle swarm optimization," IEEE International Power Electronics and Motion Control Conference, pp.63-66,2010.

[12]. S. Palanki and S. Kolavennu, "Simulation of control of a CSTR Process," International Journal of Engineering, vol.19, no. 3, pp.398-402,2003.

[13]. J. Kennedy and R. Eberhart, "Particle swarm Optimization" Proc. IEEE International Conference on Nueral Networks, vol.4, pp. 1942-1948,1995.

[14]. B. Nagaraj and P. Vijayakumar, "A comparative study of PID controller tuning using GA, EP, PSO, and ACO," Journal of Automation, Mobile Robotics \& Intelligent systems, vol. 5, no. 2,pp. 42-48,2011. 